\title{
Extracting the hysteresis loops of magnetic interfaces from optical second-harmonic intensity measurements
}

\author{
J F McGilp, L Carroll and K Fleischer \\ School of Physics, Trinity College Dublin, Dublin 2, Republic of Ireland \\ E-mail: jmcgilp@tcd.ie
}

Received 25 June 2007, in final form 12 July 2007

Published 3 September 2007

Online at stacks.iop.org/JPhysCM/19/396002

\begin{abstract}
Surface- and interface-sensitive optical techniques, such as optical secondharmonic generation (SHG), allow the buried interfacial structure of centrosymmetric materials to be explored through thin capping layers, and magnetic SHG (MSHG) extends this to magnetic interfaces. However, the variation of the MSHG intensity with magnetic field does not measure hysteresis loops directly, because the loops are displaced by an amount dependent on the crystallographic response and its phase difference with respect to the magnetic response, and also because there is a quadratic magnetization contribution to the SH intensity that may be significant. Two new procedures are reported for extracting hysteresis loops directly from the MSHG intensity. The first is applicable to all magnetic interfaces, including exchange-biased structures, where the saturation magnetization for positive and negative magnetic fields is equal and opposite. The second applies to all centrosymmetric hysteresis loops. These procedures correct for the quadratic response, allowing experimental geometries to be chosen that maximize the magnetic contribution, thus improving the signal-to-noise ratio and the sensitivity of the technique.
\end{abstract}

(Some figures in this article are in colour only in the electronic version)

\section{Introduction}

Low-dimensional magnetic systems, such as ultra-thin ferromagnetic films or multi-layer stacks of ferromagnetic and antiferromagnetic films, exhibit interesting physical phenomena not observed in bulk magnetic systems. The magnetic properties of such low-dimensional systems often depend sensitively on both the morphology and symmetry of the films and the substrate on which they are grown [1]. Magnetic nanostructures must be protected from the environment if they are to have a technological application and this is often accomplished by capping the structure with a thin non-magnetic layer. However, capping these films with protective layers of non-magnetic material may modify their magnetic properties [2], making the characterization 
of the buried interfacial region of such a capped structure essential to understanding the underlying physics and materials science. Epioptic techniques, such as optical secondharmonic generation (SHG), allow the buried interfacial structure of centrosymmetric materials to be explored through thin capping layers, where conventional surface characterization techniques are ineffective [3]. Magnetic second-harmonic generation (MSHG) extends this technique to the exploration of magnetic surfaces and interfaces $[4,5]$, allowing the lowdimensional magnetization of buried interfaces to be examined. As discussed in detail below, the MSHG intensity from such a magnetized interface may be expressed as

$$
\mathbf{I}(2 \omega ; \mathbf{H}) \propto\left|\chi_{\text {even }}^{\text {eff }} \mathbf{E}(\omega) \mathbf{E}(\omega)+\chi_{\text {odd }}^{\text {eff }} \mathbf{E}(\omega) \mathbf{E}(\omega) \mathbf{M}(\mathbf{H})\right|^{2}
$$

where $\chi_{\text {even }}^{\text {eff }}$ is the effective third-rank crystallographic susceptibility tensor, $\mathbf{E}(\omega)$ is the fundamental electric field strength, $\chi_{\text {odd }}^{\text {eff }}$ is the effective fourth-rank axial magnetic susceptibility tensor, $\mathbf{M}$ is the magnetization and $\mathbf{H}$ is the applied magnetic field. The number of non-zero tensor components depends on the composition, crystallographic and magnetic symmetry of the medium, and the number of these components accessed in a particular experiment depends on the sample orientation and the optical polarization geometry.

While it is now well established that the nonlinear magneto-optic effect may be orders of magnitude larger than the linear magneto-optic effect $[6,7]$, the crystallographic response in the above equation remains, in general, substantially larger than the magnetic response, allowing the quadratic term in the nonlinear response to be neglected. However, the signalnoise ratio (SNR) of hysteresis loops deduced from such MSHG measurements is inherently limited because the intensity is dominated by the non-magnetic crystallographic contributions. This is most clearly seen for centrosymmetric loops, where equation (10) shows that the loop is extracted directly from the difference in intensities arising from the change in the magnetic contribution. For some magnetic interfaces, careful choice of experimental geometry allows the crystallographic contribution to be reduced such that it is comparable to the magnetic contribution, resulting in a significantly improved SNR. However, when this condition is valid the MSHG intensity is no longer linearly related to the magnetization of the interface, as has been discussed recently in interpreting MSHG studies of exchange-biased $\mathrm{CoO} / \mathrm{Fe}$ multilayers [8]. Optimizing the SNR of the MSHG measurement by reduction of the crystallographic contribution may lead to the breakdown of the simple linear relationship between the MSHG intensity and the interfacial magnetization under investigation.

Two new procedures are described below that allow the hysteresis loops of a magnetic interface to be deduced from MSHG measurements where the quadratic response is significant. A hysteresis loop is a complex, nonlinear, nonequilibrium, nonlocal phenomenon and only a few simple curves have been calculated (using Stoner-Wohlfarth theory) [9]. However, many of the experimental loops of simple material systems are centrosymmetric, and even the more complex, acentric, exchange-bias systems show a remaining symmetry where the saturation magnetization for positive and negative magnetic fields is equal and opposite [10-16]. Some of the latter systems show a small vertical displacement of the loop of $<5 \%$ [17], which the general approach described below will not reveal. The two procedures exploit the symmetry of the systems: the general procedure is applicable to all magnetic interfaces where the saturation magnetization for positive and negative magnetic fields is equal and opposite; the second procedure is easier to implement, but applies to centrosymmetric hysteresis loops only. The two approaches account for the non-magnetic crystallographic contribution, and both the linear and quadratic magnetization contribution to the intensity, allowing the extraction of good quality hysteresis loops from the MSHG intensity. The effectiveness of the two procedures is demonstrated by applying them to a range of simulated MSHG intensity measurements, containing realistic Poissonian noise, and also to MSHG measurements of Au-capped ultra-thin 
Fe films on vicinal W(110). Although MSHG is a second-order optical process, it is shown that the SNRs of the hysteresis loops from the Fe films are comparable to those of similar films obtained using the first-order magneto-optic Kerr effect (MOKE) [18].

\section{Theory}

Within the electric dipole approximation, the nonlinear second-order polarization of a magnetic medium can be written as

$$
\mathbf{P}(2 \omega ; \mathbf{H})=\chi^{\mathbf{c}} \mathbf{E}(\omega) \mathbf{E}(\omega)+\chi^{\mathbf{m}} \mathbf{E}(\omega) \mathbf{E}(\omega) \mathbf{M}(\mathbf{H}) .
$$

Within the same approximation, for a multi-layer sample consisting of centrosymmetric materials, the non-zero tensor components originate at the surface and interfaces only. The magnetic term is assumed to depend linearly on the magnetization, allowing the individual tensor components to be expressed as [19]

$$
\chi_{i j k}\left(M_{L}\right)=\chi_{i j k}(0)+X_{i j k L} M_{L}
$$

where $\chi_{i j k}\left(M_{L}\right)$ is the total magnetization-dependent nonlinear susceptibility component, $\chi_{i j k}(0)$ is the crystallographic term, which is even in the magnetization, $X_{i j k l} M_{L}$ is the magnetic term, which is odd in the magnetization, and $M_{L}$ is the component of the magnetization along $L$. Magnetostriction effects on the crystallographic term are neglected in equation (2).

Fresnel and local-field factors are required to obtain the general expression for the $\mathrm{SH}$ intensity. These factors depend on parameters such as the angle of incidence, and the input and output polarization, but do not vary significantly with the magnetic field. For the purposes of determining hysteresis loops, where only the applied magnetic field, $H$, is varied, the Fresnel and local-field factors can be included in an effective tensor component, giving (appendix A)

$$
P_{i}\left(2 \omega, \theta ; M_{L}\right)=\left\{\chi_{i j k}^{\mathrm{eff}}(0)+X_{i j k L}^{\mathrm{eff}} M_{L}\right\} E_{j}(\omega) E_{k}(\omega)
$$

The MSHG intensity, to within a scaling factor, can be expressed as

$$
I\left(2 \omega, M_{L}\right)=\left|\chi_{\text {even }}^{\text {eff }}\right|^{2}+\left\{\left|X_{\text {odd }}^{\text {eff }}\right| M_{L}\right\}^{2}+2\left|\chi_{\text {even }}^{\text {eff }}\right|\left\{\left|X_{\text {odd }}^{\text {eff }}\right| M_{L}\right\} \cos \delta
$$

where $\delta$ is the phase difference between the even crystallographic and the odd magnetic contributions. Reversal of the magnetization thus produces a phase shift of $\pi$, which has been demonstrated experimentally for a multilayer system [20]. Equation (4) can be solved for $\left\{\left|X_{\text {odd }}^{\text {eff }}\right| M_{L}\right\}$, which is proportional to the magnetization:

$$
\left\{\left|X_{\text {odd }}\right| M\right\}=-\gamma \pm \sqrt{I(M)+\gamma^{2}-\left|\chi_{\text {even }}\right|^{2}}
$$

where eff and $L$ are dropped for convenience, $\gamma=\left|\chi_{\text {even }}\right| \cos \delta$ and the root is real. The hysteresis curve can be determined from equation (5) if $\left|\chi_{\text {even }}\right|$ and $\delta$ are known. These parameters can be evaluated directly from the MSHG intensity data for systems where the saturation magnetization, $M^{\text {sat }} \pm$, is equal and opposite when the field is reversed. For such symmetric saturation conditions it is shown in appendix A that

$$
\gamma=\frac{1}{2}\left\{\sqrt{I^{\mathrm{sat}+}-I^{\mathrm{tp}}}-\sqrt{I^{\mathrm{sat}-}-I^{\mathrm{tp}}}\right\} \quad \text { and } \quad \tan \delta=\sqrt{I^{\mathrm{tp}}} / \gamma
$$

where $I^{\text {sat }} \pm$ are the saturation intensities and $I^{\text {tp }}$ is the intensity at the turning point of the MSHG curve (see figure 1(c), left panel). Thus a quantitative extraction of the hysteresis curve is possible in situations where a turning point can be identified, and $\chi_{\text {even }}$ and $\delta$ can also be determined.

Where the magnetic contribution is small relative to the crystallographic contribution, the quadratic term in equation (4) can be neglected, giving

$$
I(M)=\left|\chi_{\text {even }}\right|^{2}+2\left|\chi_{\text {even }}\right|\left\{\left|X_{\text {odd }}\right| M\right\} \cos \delta .
$$



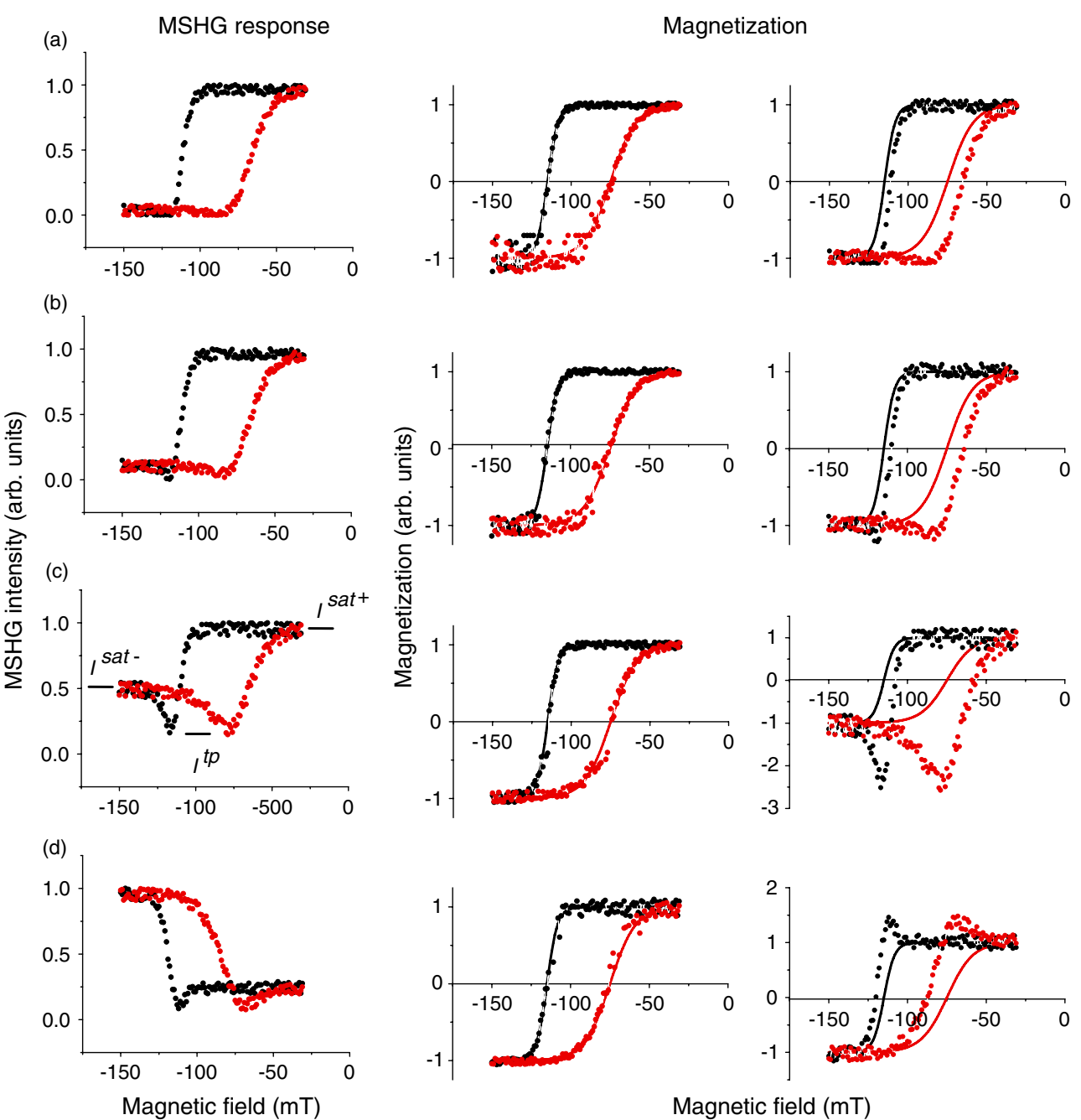

Figure 1. Left panels: simulated acentric and exchange-biased MSHG response for $\left|\chi_{\text {odd }} / \chi_{\text {even }}\right|=$ 1.5. Centre panels: extracted magnetization loops, using equations (5) and (6), compared to the actual loop (solid lines). Right panels: extracted magnetization loops, using equation (8), which neglects the quadratic term. (a) $\delta=0^{\circ}$, (b) $\delta=30^{\circ}$, (c) $\delta=70^{\circ}$, (d) $\delta=130^{\circ}$. Examples of saturation and turning point positions are shown in (c), left panel. The loop has an exchange bias of $-95 \mathrm{mT}$, with total coercivity of $40 \mathrm{mT}$.

Symmetric saturation conditions then give

$$
\left\{\left|X_{\text {odd }}\right| M\right\} \cos \delta=\frac{I(M)-\frac{1}{2}\left(I^{\mathrm{sat}+}+I^{\mathrm{sat}-}\right)}{\sqrt{2\left(I^{\mathrm{sat}+}+I^{\mathrm{sat}-}\right)}} .
$$

This expression is equivalent to previous linearized expressions [7]. Finally, there is an intermediate situation where, although the magnetic contribution is not small, the MSHG intensity does not show a turning point. In this situation, appendix A shows that a simple approximation is to set $I^{\text {tp }}=0$, giving

$$
\gamma=\frac{1}{2}\left\{\sqrt{I^{\text {sat }+}}+\sqrt{I^{\text {sat- }}}\right\} \quad \text { and } \quad\left|\chi_{\text {even }}\right|=\gamma .
$$


The above equations allow some special cases to be identified. For $\delta=\pi / 2$ and $\delta=3 \pi / 2$, $\cos \delta=0$ and the linear cross-term vanishes in equation (4), giving $I^{\text {sat- }}=I^{\text {sat }+}$ in the MSHG intensity plot. Only the quadratic term contributes to the magnetic response, preventing the determination of hysteresis curves unless the magnetic contribution is comparable to, or larger than, the crystallographic contribution. For $\delta=0$ and $\pi, I^{\text {tp }}=0$ (appendix A); the turning point, where it exists, occurs at zero intensity.

For the centrosymmetric loops often found with simple ferromagnetic and superparamagnetic interface structures, appendix B shows that

$$
M^{ \pm}(H) \propto I^{ \pm}(2 \omega, H)-I^{\mp}(2 \omega,-H)
$$

where $M^{+}(H)$ has $H$ increasing from an initial negative value, and $M^{-}(H)$ has $H$ decreasing from an initial positive value, with $I^{+}(2 \omega, H)$ and $I^{-}(2 \omega, H)$ the corresponding intensities.

\section{Experiment}

Sample preparation of the Au-capped ultra-thin Fe films is described in detail elsewhere [21]. Pseudomorphic Fe films were grown under ultra-high-vacuum conditions (base pressure $<4 \times 10^{-11}$ mbar) on a clean vicinal W(110) single-crystal substrate, offcut $1.4^{\circ}$ in the [1ํㅣㄹ direction. The Fe films were protected from ex situ contamination by a $12-16 \mathrm{~nm}$ thick capping layer of Au. The capped samples were placed in an optical cryostat and the MSHG measured at near-normal incidence, at $80 \mathrm{~K}$, as a function of applied magnetic field strength. A femtosecond laser system, tuned to $1.55 \mathrm{eV}$, was used as the fundamental frequency light source. Calculations predict a strong MSHG response from the Fe films in this spectral region [22]. Unamplified, $130 \mathrm{fs}$ Ti:sapphire laser pulses of average power of $0.9 \mathrm{~W}$ were used, at a repetition rate of $76 \mathrm{MHz}$ and with a beam size of $40 \mu \mathrm{m}$ at the sample. A good SNR was obtained for these samples when the fundamental beam was directed towards the sample surface at near-normal incidence. The input polarization was then adjusted such that it was offset by $<5^{\circ}$ from the [001] direction of the atomic steps of the vicinal W(110) surface, while the output polarizer was adjusted so that only the intensity of MSHG polarized parallel to the steps was measured. The samples were magnetized along their easy axis in the [1 10$]$ direction, which is in the sample plane orthogonal to the direction of the atomic steps.

\section{Results and discussion}

Many simple magnetic interfaces show centrosymmetric hysteresis loops, but there is considerable interest currently in more complex, exchange-biased thin film systems, some of which show acentric hysteresis loops $[10,11,13,16]$. Figures 1-3 show, in the left panels, the simulated MSHG response, with added Poissonian noise, arising from an acentric hysteresis response, exchange-biased by $-95 \mathrm{mT}$ (solid lines in centre and right panels). The shape of the loop is similar to that observed from exchange-biased, virgin $\mathrm{CoO} / \mathrm{Co}$ bilayers [12].

The left panel of figure 1 shows the response for $\left|\chi_{\text {odd }} / \chi_{\text {even }}\right|=1.5$, with various values of the phase difference, $\delta$, and noting that $\cos \delta=\cos \left(360^{\circ}-\delta\right)$. It can be seen that, as $\delta$ increases, the turning points change from being barely discernable to being well defined. For $180^{\circ}>\delta>90^{\circ}$, the curves are reflected about a vertical axis. The results of applying equations (5) and (6) are shown in the centre panel, where a local fit was used to find the positions of the turning point. In general, very good agreement with the model loop is obtained. The extracted curve is noisy where the MSHG signal is small, as is to be expected. Also, in the region of the turning point, where the square root in equation (5) is close to zero, some points may be lost where the noise renders the root imaginary. Equation (5) shows that, 

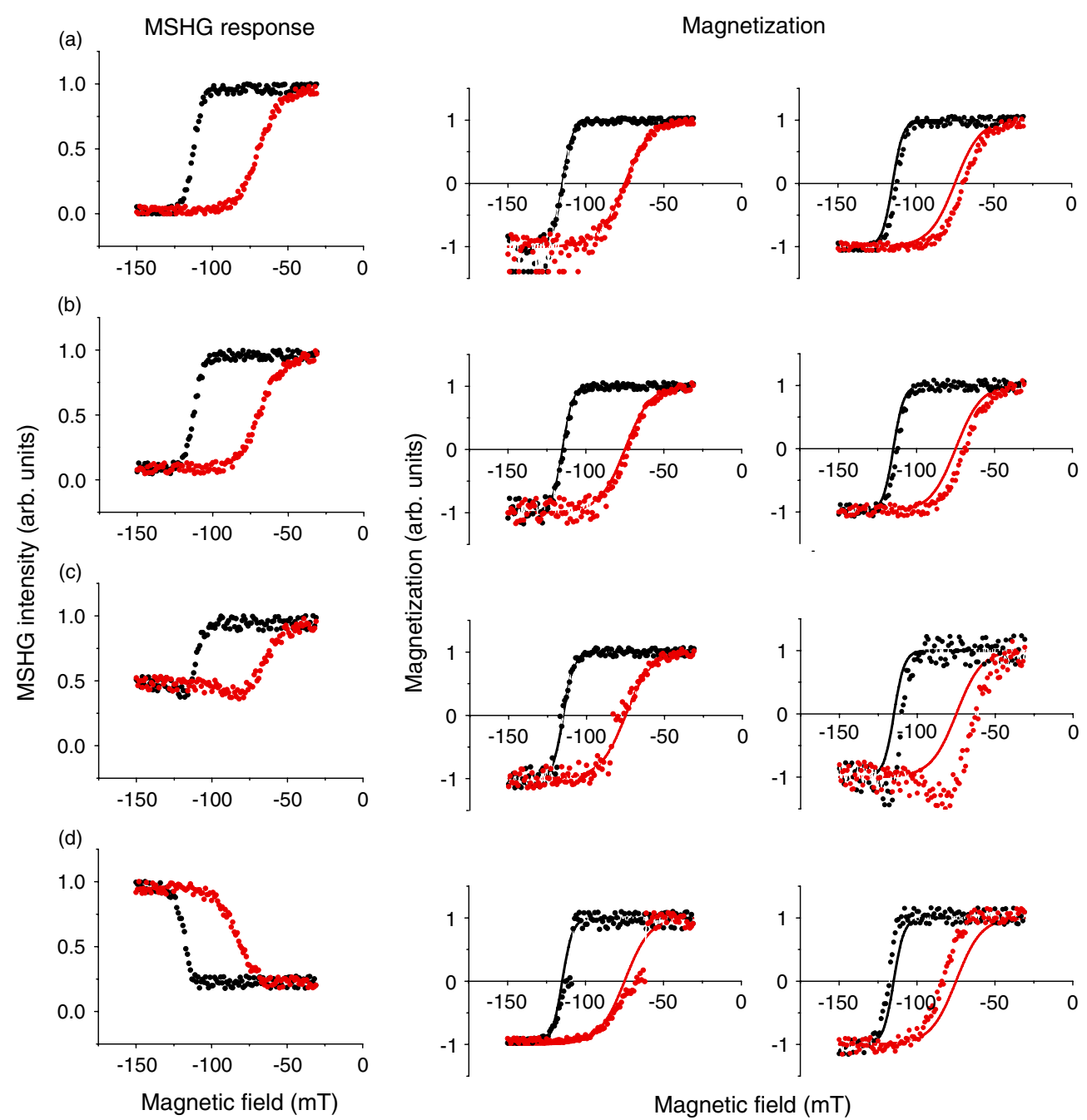

Figure 2. Left panels: simulated MSHG response for $\left|\chi_{\text {odd }} / \chi_{\text {even }}\right|=0.75$. Centre panels: extracted magnetization loops, compared to the actual loop (solid lines); (a), (b) using approximate equations (5) and (9); (c), (d) using equations (5) and (6). Right panels: extracted magnetization loops, using equation (8), which neglects the quadratic term. (a) $\delta=0^{\circ}$, (b) $\delta=30^{\circ}$, (c) $\delta=70^{\circ}$, (d) $\delta=130^{\circ}$.

where $180^{\circ}>\delta>90^{\circ}, \cos \delta$ and thus $\gamma$ changes sign, producing the reflected curves of figure 1(d). The right panel of the figure shows the results of using equation (8), which neglects the quadratic term. Even where the turning point is indistinct, the shape of the extracted loop differs significantly from the model and there is a large error in the exchange bias.

Figure 2 shows equivalent results for $\left|\chi_{\text {odd }} / \chi_{\text {even }}\right|=0.75$. The turning points in figures 2(a) and (b), left panel, could not be identified and equations (5) and (9) were used to find approximate solutions. The agreement with the model loop is very good (centre panel), while using equation (8), which neglects the quadratic term, produces a much poorer result (right panel). Turning points were found for figures 2(c) and (d), allowing the use of equations (5) and (6), but figure 2(d) shows a deviation from the model loop near zero magnetization, probably indicating an error in the fitted turning point values. 

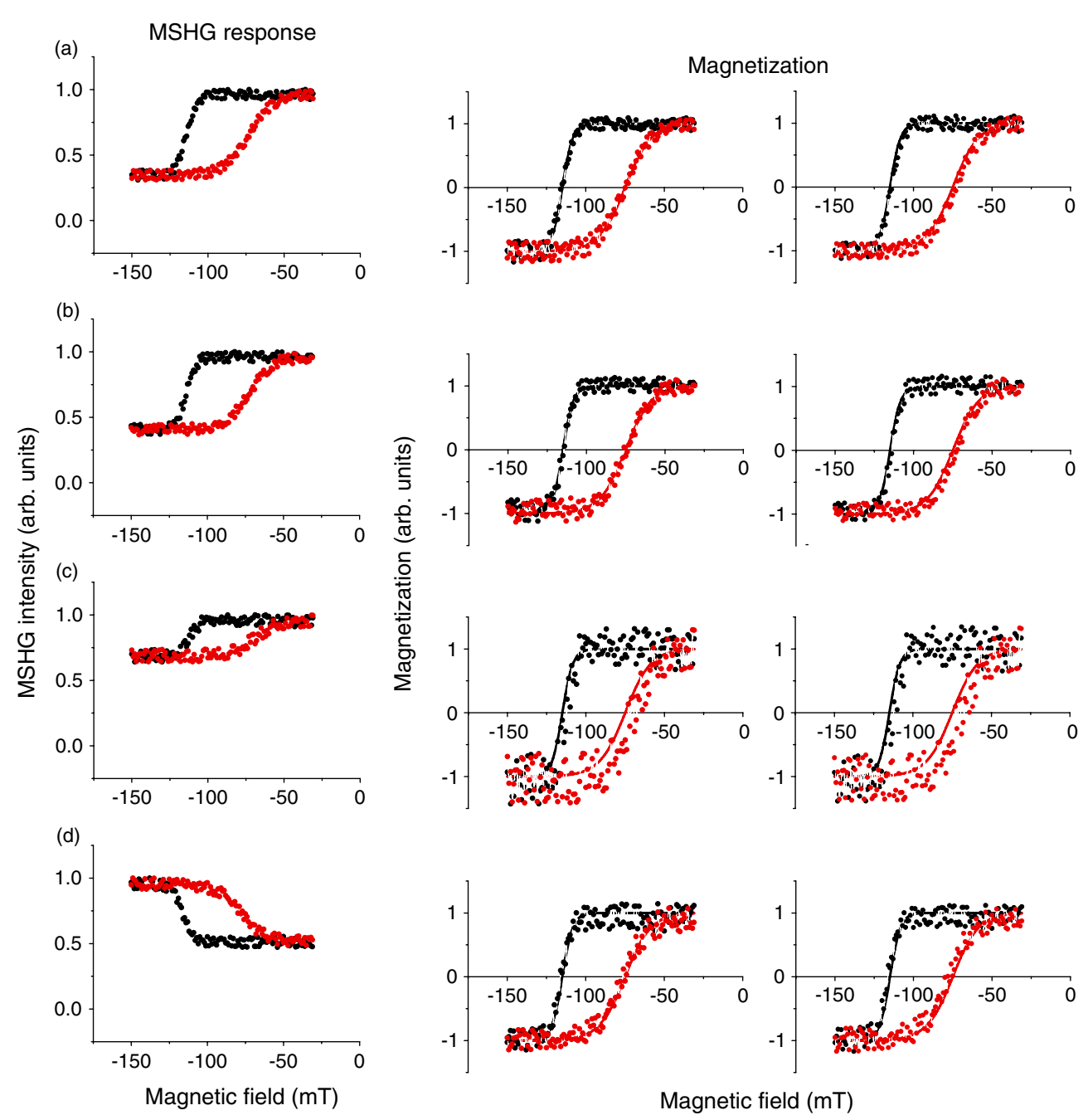

Figure 3. Left panels: simulated MSHG response for $\left|\chi_{\text {odd }} / \chi_{\text {even }}\right|=0.25$. Centre panels: extracted magnetization loops, using approximate equations (5) and (9), compared to the actual loop (solid lines). Right panels: extracted magnetization loops, using equation (8), which neglects the quadratic term. (a) $\delta=0^{\circ}$, (b) $\delta=30^{\circ}$, (c) $\delta=70^{\circ}$, (d) $\delta=130^{\circ}$.

Figure 3 shows the results for $\left|\chi_{\text {odd }} / \chi_{\text {even }}\right|=0.25$. No turning points could be identified and equations (5) and (9) were used to find approximate solutions. The agreement with the model loop is again very good (centre panel), but neglecting the quadratic term now produces only a small error in the exchange bias (right panel), as is to be expected with the reduced magnetic contribution. Figure 3(c) shows that the combination of a small magnetic contribution and a value of $\delta$ near $90^{\circ}$ results in a noisy and inaccurate hysteresis loop, if, indeed, a loop can be extracted at all.

Summarizing the simulation results for acentric loops, accurate curves can be extracted where turning points can be identified. The extracted loop then allows the exchange-bias and total coercivity fields (the width of the loop at zero magnetization), and also $\left|\chi_{\text {odd }} / \chi_{\text {even }}\right|$ and the phase shift $\delta$, to be obtained. Errors in the fields are estimated at $\leqslant 1 \mathrm{mT}$. Where the approximate approach has to be used, very good loops can be obtained with typical errors in 


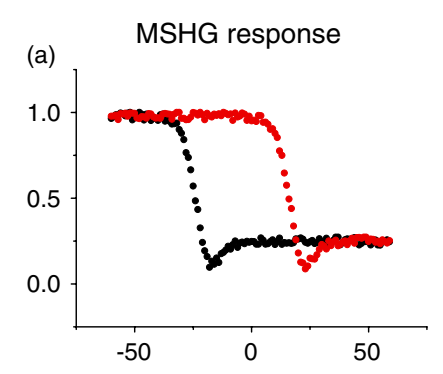

Magnetization
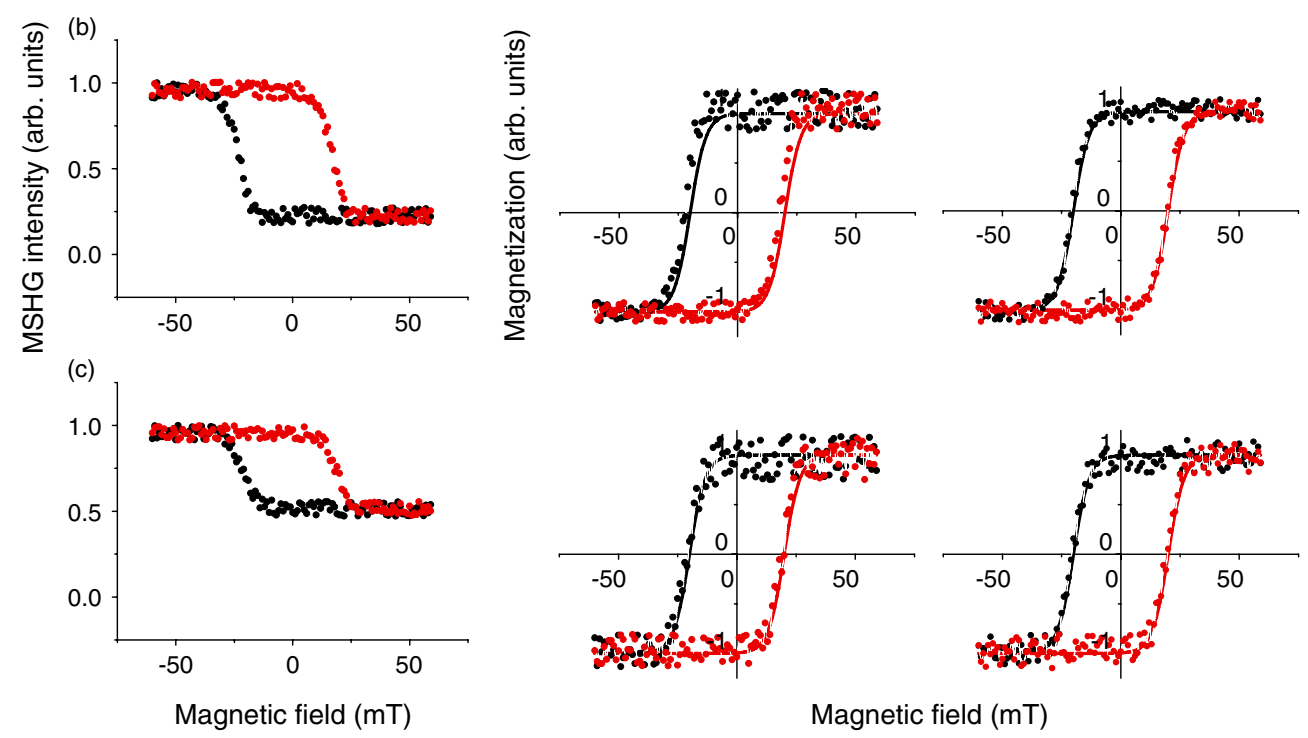

Figure 4. Left panels: simulated centrosymmetric MSHG response for $\delta=130^{\circ}$. Centre panels: extracted magnetization loops using approximate equations (5) and (9), compared with the actual loop (solid lines). Right panels: extracted magnetization loops using symmetry and equation (10). (a) $\left|\chi_{\text {odd }} / \chi_{\text {even }}\right|=1.5$, (b) $\left|\chi_{\text {odd }} / \chi_{\text {even }}\right|=0.75$, (c) $\left|\chi_{\text {odd }} / \chi_{\text {even }}\right|=0.25$.

the fields of $\leqslant 3 \mathrm{mT}$. Highly vertically compressed loops are an exception to this, the error in the exchange-bias of figure 3(c) being $7 \mathrm{mT}$. The linear approximation leads to larger errors in all cases examined.

For simpler magnetic interfaces, centrosymmetric hysteresis loops are often observed, allowing the use of equation (10) to extract the hysteresis loop. Figure 4 shows the results for $\delta=130^{\circ}$ and the previous range of values of $\left|\chi_{\text {odd }} / \chi_{\text {even }}\right|$. The centre panel shows the loops extracted using equation (5) with the approximate equation (9), while the right panel uses the exact equation (10) for centrosymmetric loops. The variation in the noise in the extracted loops reflects the different methods of extraction. Both methods produce very good results, but the simple and elegant equation (10) is clearly the preferred approach for centrosymmetric loops.

These methods are now applied to real data, obtained as described in section 3 . Figure 5 shows MSHG intensities at $80 \mathrm{~K}$, for 0.75 and 3.0 ML Fe ultra-thin films, grown on vicinal $\mathrm{W}(110)$ and capped by 12 and $16 \mathrm{~nm}$ of $\mathrm{Au}$, respectively. The MSHG response shows the offset from zero, which is determined by the relative size of the crystallographic response, $\chi_{\text {even }}$, and the phase shift, $\delta$. The suggested methodology is to apply the general approach first to determine whether the extracted loop is significantly acentric or exchange-biased. The result will then determine whether the centrosymmetric formula should be used. Figure 5(a) 

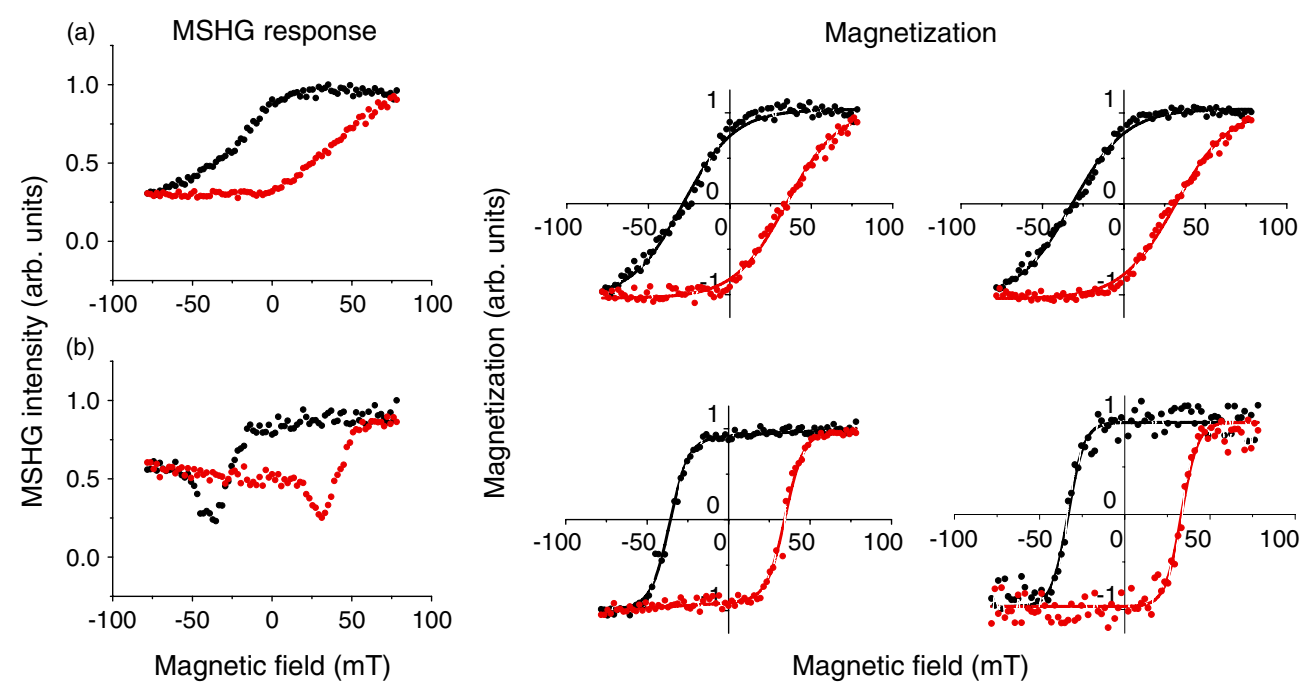

Figure 5. MSHG data for $\mathrm{W}(110) / \mathrm{Fe} / \mathrm{Au}$ nanostructures. Left panels: MSHG response for (a) $0.75 \mathrm{ML} \mathrm{Fe}$, (b) $3 \mathrm{ML}$ Fe. Centre panels: extracted magnetization loops using approximate equations (5) and (9) for (a) and equations (5) and (6) for (b). Right panels: extracted magnetization loops using symmetry and equation (10). Solid lines are fits to the extracted loops using sigmoidal curves.

shows the results for the $0.75 \mathrm{ML}$ film, where the solid lines are now fits of the extracted loops to sigmoidal functions, which allow good estimates of any exchange bias. Although close to saturation, it is possible that this loop is not fully saturated. The centre panel shows that the general approach, using equation (5) and the approximate equation (9), produces a centrosymmetric loop with a very small apparent exchange bias of $3 \pm 1 \mathrm{mT}$. This places an upper limit on the exchange bias, and is within the range of the estimated error associated with the approximate approach, discussed above. The result allows the centrosymmetric formula to be used, with the knowledge of a possible small exchange-bias error. The right panel shows the result of using equation (10), and the fit to the extracted data gives a coercivity of $32 \pm 1 \mathrm{mT}$ for the capped $0.75 \mathrm{ML}$ Fe film. The coercivity agrees well with previous Kerr effect results for this material system, although the Kerr loops were a little squarer [23].

Figure 5(b) shows the results for the 3.0 ML film. Turning points are clearly seen, allowing the exact general approach to be used. The centre panel shows the extracted hysteresis loop and the sigmoidal fits that show a centrosymmetric loop of coercivity $35 \pm 1 \mathrm{mT}$ and zero exchange bias, within error. The right panel shows the result of the direct centrosymmetric extraction, which gives a coercivity for the 3.0 ML Fe film of $33 \pm 1 \mathrm{mT}$. The general approach also gives $\left|\chi_{\text {odd }} / \chi_{\text {even }}\right|=1.90 \pm 0.02$ and a phase shift between the non-magnetic and magnetic components of $76 \pm 1^{\circ}$.

All the extracted loops have an SNR comparable to the linear magneto-optic Kerr effect (MOKE) results from vicinal $\mathrm{W}(110) / \mathrm{Fe}[18,23]$. Accounting correctly for the quadratic response allows experimental geometries to be chosen that maximize the magnetic nonlinear response in a given system, thus improving the SNR.

\section{Conclusion}

Two new procedures have been derived that allow the hysteresis loops of magnetic interfaces to be determined directly from MSHG intensity measurements in situations where the quadratic 
response is significant. The first procedure is applicable to all magnetic interfaces, including exchange-biased systems, provided the saturation magnetization for positive and negative magnetic fields is equal and opposite. The second procedure is less general, but easier to implement, and applies to all centrosymmetric hysteresis loops, including those of complex shape. These procedures correct for the crystallographic offset without the need to attempt to measure the crystallographic response separately. Both procedures account for the quadratic magnetization contributions to the intensity, allowing the extraction of good quality hysteresis loops directly from the MSHG intensity. The effectiveness of these two procedures has been demonstrated by applying them to a range of simulated MSHG intensity curves, containing realistic Poissonian noise. A methodology has been suggested whereby the general approach can be used to determine whether the extracted loops are significantly acentric or exchangebiased. If they are not, then the simpler centrosymmetric approach can be used with confidence. These procedures will not detect the small vertical shifts of the loops reported for some exchange-bias systems [17], but such shifts should not affect, significantly, the extracted loop shape or the key field parameters determined from it. The methodology has been applied to MSHG intensity measurements of Au-capped ultra-thin Fe films grown on vicinal W(110) and good quality hysteresis loops extracted. Although MSHG is a nonlinear optical process, the SNRs of the hysteresis loops from the Fe films are comparable to those of similar films obtained using the first-order magneto-optic Kerr effect (MOKE) [18]. The importance of this result is that, by correctly accounting for the quadratic response, an experimental geometry can be chosen that maximizes the magnetic nonlinear response of a particular interfacial system, thus improving the sensitivity of the technique.

\section{Acknowledgments}

This publication has emanated from research conducted with the financial support of Science Foundation Ireland, the Irish Higher Education Authority and the Irish Research Council for Science, Engineering and Technology.

\section{Appendix A. Derivation of the general expression for interfaces where the saturation magnetization for positive and negative fields is equal and opposite}

Equations (1) and (2) of section 2 require the introduction of Fresnel and local-field factors to obtain the general expression for the SH intensity. These factors depend on parameters such as the angle of incidence, and the input and output polarization, but do not vary significantly with the magnetic field (linear magneto-optic effects are very small). Equations (1) and (2) then give

$P_{i}\left(2 \omega, \theta ; M_{L}\right)=f_{i j k}(\omega, 2 \omega, \theta) L_{i i}(2 \omega) L_{j j}(\omega) L_{k k}(\omega)\left\{\chi_{i j k}(0)+X_{i j k L} M_{L}\right\} E_{j}(\omega) E_{k}(\omega)$

where $f_{i j k}(\omega, 2 \omega, \theta)$ is a Fresnel factor for angle of incidence $\theta$, and $L_{i i}(2 \omega)$ is a local-field factor [24]. For the purposes of determining hysteresis loops, where only the applied magnetic field, $H$, is varied, the Fresnel and local-field factors can be included in an effective tensor component, giving

$$
P_{i}\left(2 \omega, \theta ; M_{l}\right)=\left\{\chi_{i j k}^{\mathrm{eff}}(0)+X_{i j k L}^{\mathrm{eff}} M_{L}\right\} E_{j}(\omega) E_{k}(\omega) .
$$

The input and output polarizations, and the magnetization direction, will determine values of $i, j, k$ and $L$ and are fixed in a given hysteresis loop measurement. The MSHG intensity 
now can be written, within a scaling factor, as

$$
I\left(2 \omega, \pm M_{L}\right)=\left|\chi_{\text {even }}^{\text {eff }}\right|^{2}+\left|X_{\text {odd }}^{\text {eff }} M_{L}\right|^{2} \pm 2\left|\chi_{\text {even }}^{\text {eff }}\right|\left|X_{\text {odd }}^{\text {eff }} M_{L}\right| \cos \delta
$$

where $\delta$ is the phase difference between the even crystallographic and the odd magnetic contributions. The effective tensor components of equation (A.2) for the particular interfacial structure and experimental geometry have been summed to obtain a total $\chi_{\text {even }}^{\text {eff }}$ and $X_{\text {odd }}^{\text {eff }}$, which now include the outgoing Fresnel coefficients at frequency $2 \omega$. Reversal of the magnetization produces a phase shift of $\pi$, which has been demonstrated experimentally for a multilayer system [20]. As $\left|X_{\text {odd }}^{\text {eff }} M_{L}\right|=\left|X_{\text {odd }}^{\text {eff }}\right|\left|M_{L}\right|$ and $M_{L}$ is real, equation (A.3) can be re-expressed as

$$
I\left(2 \omega, M_{L}\right)=\left|\chi_{\text {even }}^{\text {eff }}\right|^{2}+\left\{\left|X_{\text {odd }}^{\text {eff }}\right| M_{L}\right\}^{2}+2\left|\chi_{\text {even }}^{\text {eff }}\right|\left\{\left|X_{\text {odd }}^{\text {eff }}\right| M_{L}\right\} \cos \delta
$$

to within an arbitrary scaling factor. Equation (A.4) can be solved for $\left\{\left|X_{\text {odd }}^{\text {eff }}\right| M_{L}\right\}$, which is proportional to the magnetization:

$$
\begin{aligned}
\left\{\left|X_{\text {odd }}\right| M\right\} & =-\left|\chi_{\text {even }}\right| \cos \delta \pm \sqrt{I(M)+\left|\chi_{\text {even }}\right|^{2} \cos ^{2} \delta-\left|\chi_{\text {even }}\right|^{2}} \\
& =-\gamma \pm \sqrt{I(M)+\gamma^{2}-\left|\chi_{\text {even }}\right|^{2}}
\end{aligned}
$$

where eff and $L$ are dropped for convenience, $\gamma=\left|\chi_{\text {even }}\right| \cos \delta$, and the root is always real $\left(I(M)+\gamma^{2}-\left|\chi_{\text {even }}\right|^{2}=\left(\left\{\left|X_{\text {odd }}\right| M\right\}+\gamma\right)^{2}>0\right)$. The magnetization varies continuously with the applied magnetic field, $H$, and differentiation of equation (A.5) with respect to $H$ allows a turning point (tp) to be identified:

$\mathrm{d} I(M) / \mathrm{d} H=2\left|\chi_{\text {even }}\right|\left\{\left|X_{\text {odd }}\right|(\mathrm{d} M / \mathrm{d} H)\right\} \cos \delta+2\left\{\left|X_{\text {odd }}\right|^{2} M(\mathrm{~d} M / \mathrm{d} H)\right\}=0$.

If the hysteresis curve is sigmoidal, $(\mathrm{d} M / \mathrm{d} H) \neq 0$ for $|M|<\left|M^{\text {sat }}\right|$ and can be cancelled (care will be required with more complicated hysteresis curves), giving

$$
\left\{\left|X_{\text {odd }}\right| M\right\}^{\mathrm{tp}}=-\left|\chi_{\text {even }}\right| \cos \delta=-\gamma \quad \text { and, from (A.4), } \quad I^{\mathrm{tp}}=\left|\chi_{\text {even }}\right|^{2}-\gamma^{2} .
$$

Equation (A.5) then shows that the value of the root is zero at the turning point, where its contribution changes sign. If the magnetic contribution is small relative to the crystallographic response, equation (A.6) shows that a turning point may not exist.

The hysteresis curve can be determined from equation (A.5) if $\left|\chi_{\text {even }}\right|$ and $\delta$ are known. These parameters can be evaluated directly from the MSHG intensity data for systems where the saturation magnetization, $M^{\text {sat }} \pm$, is equal and opposite when the field is reversed. For such symmetric saturation conditions,

$$
\begin{aligned}
& \left\{\left|X_{\text {odd }}\right| M^{\text {sat }+}\right\}=-\gamma+\sqrt{I^{\mathrm{sat}+}-I^{\mathrm{tp}}} \\
& \left\{\left|X_{\text {odd }}\right| M^{\mathrm{sat}-}\right\}=-\gamma-\sqrt{I^{\mathrm{sat}-}-I^{\mathrm{tp}}}=-\left\{\left|X_{\text {odd }}\right| M^{\mathrm{sat}+}\right\}
\end{aligned}
$$

giving

$\gamma=\frac{1}{2}\left\{\sqrt{I^{\mathrm{sat}+}-I^{\mathrm{tp}}}-\sqrt{I^{\mathrm{sat}-}-I^{\mathrm{tp}}}\right\} \quad$ and $\quad \tan \delta=\sqrt{I^{\mathrm{tp}}} / \gamma$.

Where the odd contribution is very small relative to the even contribution, the quadratic term in equation (A.4) can be neglected, giving

$$
I(M)=\left|\chi_{\text {even }}\right|^{2}+2\left|\chi_{\text {even }}\right|\left\{\left|X_{\text {odd }}\right| M\right\} \cos \delta .
$$

Symmetric saturation conditions then give

$$
\begin{aligned}
& \left\{\left|X_{\text {odd }}\right| M\right\}^{\text {sat }+}=\left(I^{\text {sat }+}-\left|\chi_{\text {even }}\right|^{2}\right) / 2\left|\chi_{\text {even }}\right| \cos \delta \\
& \left\{\left|X_{\text {odd }}\right| M\right\}^{\text {sat }-}=\left(I^{\text {sat }-}-\left|\chi_{\text {even }}\right|^{2}\right) / 2\left|\chi_{\text {even }}\right| \cos \delta=-\left\{\left|X_{\text {odd }}\right| M\right\}^{\text {sat }+}
\end{aligned}
$$

and $\left|\chi_{\text {even }}\right|^{2}=\frac{1}{2}\left(I^{\mathrm{sat}+}+I^{\mathrm{sat}-}\right)$. 
Thus,

$$
\left\{\left|X_{\text {odd }}\right| M\right\} \cos \delta=\frac{I(M)-\frac{1}{2}\left(I^{\mathrm{sat}+}+I^{\mathrm{sat}-}\right)}{\sqrt{2\left(I^{\mathrm{sat}+}+I^{\mathrm{sat}-}\right)}} .
$$

Finally, there is an intermediate situation where, although the magnetic contribution is not small, the MSHG intensity does not show a turning point. In this situation

$$
I^{\text {sat- }}=\left|\chi_{\text {even }}\right|^{2}+2\left|\chi_{\text {even }}\right|\left\{\left|X_{\text {odd }}\right| M^{\text {sat- }}\right\} \cos \delta+\left\{\left|X_{\text {odd }}\right| M^{\text {sat }-}\right\}^{2}
$$

and, from equation (A.6), $I^{\mathrm{tp}}=\left|\chi_{\mathrm{even}}\right|^{2}\left(1-\cos ^{2} \delta\right)$, giving

$$
\begin{gathered}
I^{\text {sat }-}=I^{\mathrm{tp}} \\
+\left|\chi_{\text {even }}\right|^{2} \cos ^{2} \delta+2\left|\chi_{\text {even }}\right|\left\{\left|X_{\text {odd }}\right| M^{\text {sat }-}\right\} \cos \delta+\left\{\left|X_{\text {odd }}\right| M^{\text {sat }-}\right\}^{2} \\
=I^{\mathrm{tp}}+\left(\left|\chi_{\text {even }}\right| \cos \delta+\left\{\left|X_{\text {odd }}\right| M^{\text {sat }-}\right\}\right)^{2} .
\end{gathered}
$$

Equation (A.10) shows that $I^{\text {sat- }} \geqslant I^{\text {tp }} \geqslant 0$, the intensity at the turning point always lying below the negative saturation intensity. This suggests that the simplest approximation is to set $I^{\text {tp }}=0$, giving

$$
\gamma=\frac{1}{2}\left\{\sqrt{I^{\text {sat }+}}+\sqrt{I^{\text {sat }}}\right\} \quad \text { and } \quad\left|\chi_{\text {even }}\right|=\gamma
$$

where, in the absence of a turning point, only the positive root of equation (A.5) exists, leading to a summation, in contrast to equation (A.7), where a difference was obtained.

\section{Appendix B. Derivation for systems where the hysteresis loop is centrosymmetric}

The MSHG intensity is given by

$$
I(2 \omega, H)=\left|\chi_{\text {even }}+\chi_{\text {odd }} M(H)\right|^{2}
$$

where the dependence of the magnetization, $M$, on the magnetic field, $H$, is now shown explicitly. Both the MSHG intensity and the magnetization are assumed to comprise two curves: right-hand curves, $I^{+}(2 \omega, H)$ and $M^{+}(H)$, where $H$ is increasing from an initial negative value, and left-hand curves, $I^{-}(2 \omega, H)$ and $M^{-}(H)$, where $H$ is decreasing from an initial positive value. Equation (A.4) for the two curves can be re-expressed as

$I^{ \pm}(2 \omega, H)=\left|\chi_{\text {even }}\right|^{2}+\left\{\left|X_{\text {odd }}\right| M^{ \pm}(H)\right\}^{2}+2\left|\chi_{\text {even }}\right|\left\{\left|X_{\text {odd }}\right| M^{ \pm}(H)\right\} \cos \delta$.

For interfaces where the hysteresis loops are centrosymmetric, $M^{+}(H) \equiv-M^{-}(-H)$. Applying this identity to equation (B.2) for the two curves,

$I^{ \pm}(2 \omega, H)=\left|\chi_{\text {even }}\right|^{2}+\left\{\left|X_{\text {odd }}\right| M^{\mp}(-H)\right\}^{2}-2\left|\chi_{\text {even }}\right|\left\{\left|X_{\text {odd }}\right| M^{\mp}(-H)\right\} \cos \delta$.

It follows from equation (B.3) that

$I^{\mp}(2 \omega,-H)=\left|\chi_{\text {even }}\right|^{2}+\left\{\left|X_{\text {odd }}\right| M^{ \pm}(H)\right\}^{2}-2\left|\chi_{\text {even }}\right|\left\{\left|X_{\text {odd }}\right| M^{ \pm}(H)\right\} \cos \delta$.

The difference of equations (B.2) and (B.4) is

$$
I^{ \pm}(2 \omega, H)-I^{\mp}(2 \omega,-H)=4\left|\chi_{\mathrm{even}}\right|\left\{\left|X_{\mathrm{odd}}\right| M^{ \pm}(H)\right\} \cos \delta
$$

and thus

$$
M^{ \pm}(H) \propto I^{ \pm}(2 \omega, H)-I^{\mp}(2 \omega,-H)
$$




\section{References}

[1] Bader S D 2002 Surf. Sci. 500172

[2] Weber W, Kerkmann D, Pescia D, Wesner D A and Guntherodt G 1990 Phys. Rev. Lett. 652058

[3] McGilp J F 1995 Prog. Surf. Sci. 491

[4] Ru-Pin P, Wei H D and Shen Y R 1989 Phys. Rev. B 391229

[5] Reif J, Zink C, Schneider C M and Kirschner J 1991 Phys. Rev. Lett. 672878

[6] Koopmans B, Koerkamp M G, Rasing T and van den Berg H 1995 Phys. Rev. Lett. 743692

[7] Straub M, Vollmer R and Kirschner J 1996 Phys. Rev. Lett. 77743

[8] Valev V K, Gruyters M, Kirilyuk A and Rasing T 2005 Phys. Status Solidi b 2423027

[9] Skomski R 2003 J. Phys.: Condens. Matter 15841

[10] Fitzsimmons M R, Yashar P, Leighton C, Schuller I K, Nogues J, Nogues J, Majkrzak C F and Dura J A 2000 Phys. Rev. Lett. 843986

[11] Stiles M D and McMichael R D 2001 Phys. Rev. B 63064405

[12] Radu F, Etzkorn M, Schmitte T, Siebrecht R, Schreyer A, Westerholt K and Zabel H 2002 J. Magn. Magn. Mater. 240251

[13] Girgis E, Portugal R D, Loosvelt H, Van Bael M J, Gordon I, Malfait M, Temst K, Van Haesendonck C, Leunissen L H A and Jonckheere R 2003 Phys. Rev. Lett. 91187202

[14] Sampaio L C, Mougin A, Ferre J, Georges P, Brun A, Bernas H, Poppe S, Mewes T, Fassbender J and Hillebrands B 2003 Europhys. Lett. 63819

[15] Sampaio L C, Hamrle J, Mougin A, Ferre J, Garcia F, Fettar F, Dieny B and Brun A 2004 Phys. Rev. B 70104403

[16] Camarero J, Sort J, Hoffmann A, Garcia-Martin J M, Dieny B, Miranda R and Nogues J 2005 Phys. Rev. Lett. 95057204

[17] Nogués J, Leighton C and Schuller I K 2000 Phys. Rev. B 611315

[18] Hauschild J, Elmers H J and Gradmann U 1998 Phys. Rev. B 57 R677

[19] Kirilyuk A and Rasing T 2005 J. Opt. Soc. Am. B 22148

[20] Stolle R, Veenstra K J, Manders F, Rasing T, van den Berg H and Persat N 1997 Phys. Rev. B 55 R4925

[21] Fleischer K, Carroll L, Smith C and McGilp J F 2007 J. Phys.: Condens. Matter 19266003

[22] Dewitz J P, Jian C and Hubner W 1998 Phys. Rev. B 585093

[23] Pratzer M and Elmers H J 2003 Phys. Rev. B 6794416

[24] Shen Y R 1984 The Principles of Nonlinear Optics (New York: Wiley) 